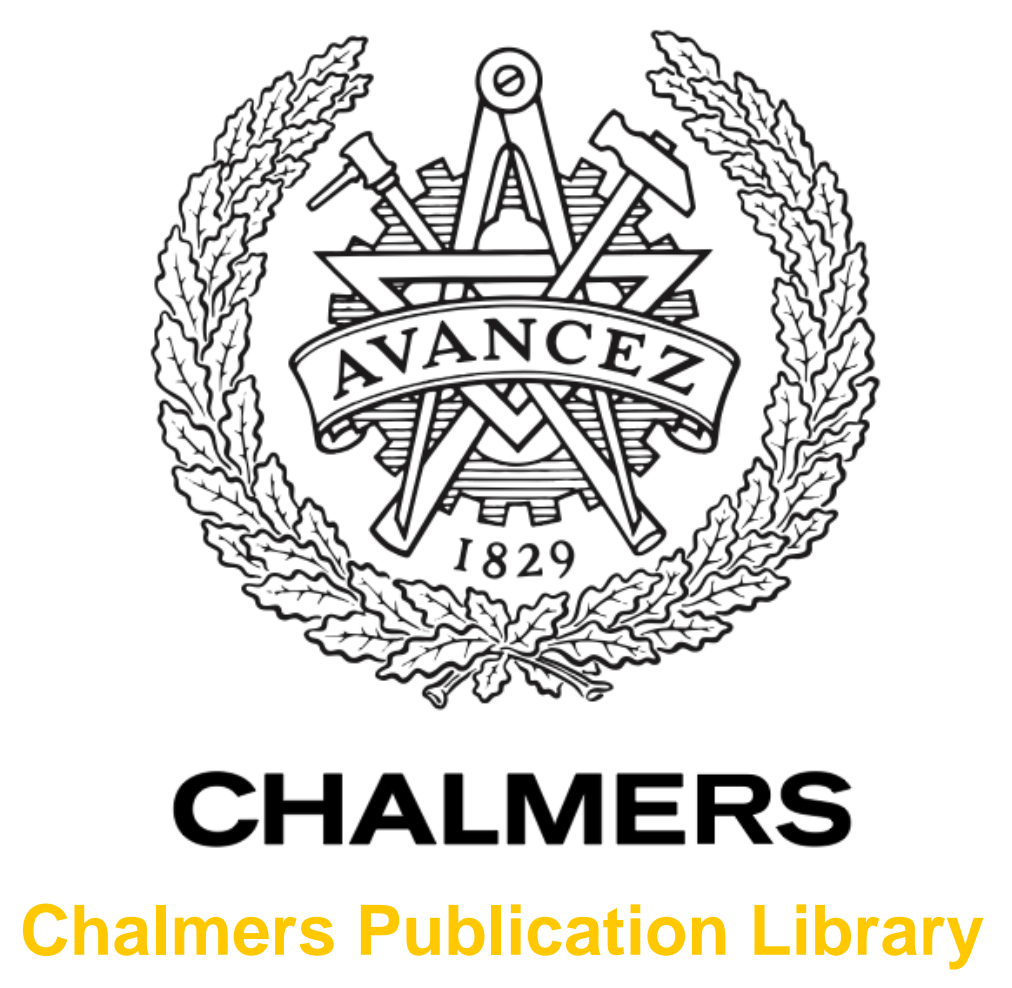

\title{
Threshold Receiver Model for Throughput of Wireless Devices with MIMO and Frequency Diversity Measured in Reverberation Chamber
}

This document has been downloaded from Chalmers Publication Library $(\mathrm{CPL})$. It is the author's version of a work that was accepted for publication in:

IEEE Antennas and Propagation Wireless Letters (ISSN: 1536-1225)

\author{
Citation for the published paper: \\ Kildal, P. ; Hussain, A. ; Chen, X. (2011) "Threshold Receiver Model for Throughput of \\ Wireless Devices with MIMO and Frequency Diversity Measured in Reverberation \\ Chamber". IEEE Antennas and Propagation Wireless Letters, vol. 10 pp. 1201-1204.
}

http://dx.doi.org/10.1109/LAWP.2011.2172909

Downloaded from: http://publications.lib.chalmers.se/publication/153310

Notice: Changes introduced as a result of publishing processes such as copy-editing and formatting may not be reflected in this document. For a definitive version of this work, please refer to the published source. Please note that access to the published version might require a subscription. 


\title{
Threshold Receiver Model for Throughput of Wireless Devices with MIMO and Frequency Diversity Measured in Reverberation Chamber
}

\author{
Per-Simon Kildal, Fellow, IEEE, Ahmed Hussain, Xiaoming Chen, Charlie Orlenius, Anton Skårbratt, \\ John Åsberg, Tommy Svensson, Thomas Eriksson
}

\begin{abstract}
We present a simple theoretical model for the throughput data rate of a wireless LTE device including the improvements of data rate due to diversity in frequency (OFDM) and spatial domains (MIMO) under frequency selective fading. The model is based on defining an ideal threshold receiver for the Line-Of-Sight (LOS) case, corresponding to reception with advanced error correcting codes. The theoretical throughput model is in agreement with measurements in a reverberation chamber of a commercial LTE device for the 1x2 SIMO case, both regarding diversity and MIMO array gains, and it can therefore be used to complement measured results in evaluation of performance of LTE devices.
\end{abstract}

Index Terms- MIMO, multipath, OFDM, OTA measurements, Rayleigh fading, reverberation chamber

\section{INTRODUCTION}

$\mathrm{T}$ HE reverberation chamber has its background in EMC testing, but its appropriateness for accurate Over-The-Air (OTA) tests of wireless devices was evident when it was shown that it represents a rich isotropic multipath environment [1], provided it is large enough and with efficient stirring methods. Its performance is based on well accepted theories [2], and it has already shown its ability to accurately measure efficiency, diversity gain [3] and maximum available capacity [4] of multi-port - more commonly called MIMO (Multiple Input Multiple Output) - antenna systems, as well as radiated power and receiver sensitivity of mobile terminals [5]. Recently, it has also been shown that the reverberation chamber can be used to measure throughput data rate of LTE and IEEE 802.11n WiFi wireless systems with OFDM and MIMO capability [6]. The Orthogonal Frequency-Division Multiplexing (OFDM) is a way of achieving diversity in the frequency domain, and MIMO can be used to realize diversity in the spatial domain, both being able to mitigate the problems

Manuscript submitted in August, 2011. This work was supported in part by the Swedish Foundation for Strategic Research (SSF) via the CHARMANT antenna systems research center at Chalmers, and by The Swedish Governmental Agency for Innovation Systems (VINNOVA) within the VINN Excellence Center Chase.

P.-S. Kildal, A. Hussain, X. Chen, T. Svensson, T. Eriksson are with Chalmers University of Technology (Chalmers), Gothenburg, Sweden (email: per-simon.kildal@chalmers.se). C. Orlenius, A. Skårbratt and J. Åsberg are with Bluetest AB. with appearance of low signal levels due to fading.

The previous measurements agree well with theoretical models for diversity gain [3, 7, 8] and MIMO capacity [4], including also agreement with alternative measurement approaches in anechoic chambers [9]. However, there exists no simple theoretical model for measured throughput data rate. Therefore, it is the purpose of the present paper to introduce a simple theoretical model for calculating throughput data rate of practical wireless device in systems with OFDM and MIMO capabilities. The theoretical results will be compared with measured results in a reverberation chamber, in order to explain dependences on embedded efficiencies of the elements of the MIMO receive antenna array of the device, the correlation between the MIMO array ports, and the effect of OFDM and its dependence on the coherence bandwidth of the environment. It is known from previous studies $[10,11]$ how to control coherence bandwidth in reverberation chambers.

It has also been shown elsewhere that bit error rates (BER) can be measured in reverberation chambers [5], and that they will become similar to those measured in real-life environments if the chamber is appropriately loaded [12].

\section{IDEAL THRESHOLD RECEIVER REPRESENTING ERROR CORRECTING CODES IN LOS}

In advanced wireless systems the bits are gathered in groups named frames, blocks or packages that are provided with Forward Error Corrections (FEC) so that the bit group becomes less sensitive to errors at the receive side than the single bit is, and it is possible to detect bit errors within each bit group and correct the error or retransmit the group. The performance of such receivers is measured in terms of a Frame Error Rate (FER), Block Error Rate (BLER) or Package Error Rate (PER) rather than Bit Error Rate (BER), and the shape of the corresponding error rate curve as a function of signal power is much steeper than the BER curve of the simplest digital receivers without FEC. We will in the continuation refer to such FER, BLER or PER as a Group Error Rate (GER). The error rate is in particular very steep in a static AWGN (Additive White Gaussian Noise) channel, i.e. a channel without fading and multipath so that there is no other distortion than AWGN. We may also refer to the AWGN channel as a pure Line-Of-Sight (LOS) channel. With modern FEC codes the above-mentioned change of the GER is very 
abrupt, from almost $100 \%$ to almost $0 \%$ at a specific signal threshold level, and that has stimulated us to define an ideal error correction receiver, for convenience and simplicity, by the following GER curve

$$
\operatorname{GER}_{\text {ideal }}(P)= \begin{cases}1 & \text { when } P<P_{t} \\ 0 & \text { when } P>P_{t}\end{cases}
$$

where $P$ is the received signal power, and $P_{t}$ is the threshold level. The throughput data rate can, when dealing with error correction codes, simply be expressed as

$$
\text { Tput }=\text { Maxrate } *\{1-G E R(P)\}
$$

where Maxrate is the data rate given by system parameters. This formula is valid both for the GER in a static LOS channel versus constant signal power $P$ and for the average GER versus average signal power $P_{a v}$ in a dynamic fading environment. The validity of the threshold receiver approximation can readily be seen from the measured LOS throughput curves in Figure 1, which have very clear threshold values. The relative throughput actually increases from 0.1 to 0.9 when the signal power increases by about $0.4 \mathrm{~dB}$.
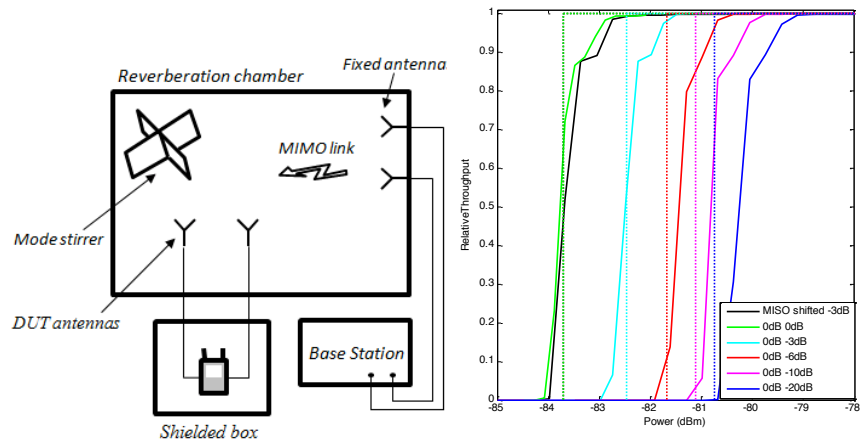

Figure 1. Left: Setup with reverberation chamber for measuring data throughput. Right: Measured (solid lines) and theoretical (dashed lines) relative throughput data rate of a commercial LTE USB device, for static LOS case, for one 2xl and several 1x2 MIMO cases, the latter with different attenuations (efficiencies) on the two ports, see Sec. V.A..

\section{AVERAGE ERROR RATE WITH THRESHOLD RECEIVER IN DYNAMIC RAYLEIGH CHANNEL WITH FLAT FADING}

In a dynamic channel with time-varying fading the GER will change with time, and therefore it is common in this case to instead deal with GER averaged over sufficiently long fading time. When the average GER has stabilized, we can refer to it as the ergodic GER for the given average signal power level $P_{a v}$. Thus, average GER value is function of $P_{a v}$.

The relation between the static LOS GER and average GER functions, referred to as $\operatorname{GER}_{L O S}(P)$ and $\operatorname{GER}_{a v}\left(P_{a v}\right)$, respectively, are given by

$$
\operatorname{GER}_{a v}\left(P_{a v}\right)=\int_{0}^{\infty} G E R_{L O S}(P) P D F\left(P / P_{a v}\right) d P
$$

where $\operatorname{PDF}\left(P / P_{a v}\right)$ is the probability density of the fading power level $P$ at the receiver input, and $P_{a v}$ is the average value of $P$. For Rayleigh fading the PDF is the exponential distribution. Note that for (3) to be valid we have to require that the signal bandwidth is much smaller than the coherence bandwidth, i.e. what is known as frequency-flat fading.

By combining (1) and (3), and using (2), we get simply

$$
\begin{aligned}
& \operatorname{GER}_{a v}\left(P_{a v}\right)=\int_{0}^{P_{t}} P D F\left(P / P_{a v}\right) d P=\operatorname{CDF}\left(P_{t} / P_{a v}\right) \\
& \text { Tput }=\text { Maxrate }^{*} \operatorname{CCDF}\left(P_{t} / P_{a v}\right)
\end{aligned}
$$

where CCDF is the complementary $\mathrm{CDF}$ and $\mathrm{CDF}$ the cumulative distribution function of the channel power. We see that for the ideal threshold receiver the relative throughput data rate is equal to the CCDF of the power distribution of the fading channel at the threshold level.

\section{ADDING DIVERSITY TO THRESHOLD RECEIVER IN DYNAMIC RAYLEIGH CHANNEL: OFDM AND MIMO}

The simple relation between the relative throughput and the CCDF of the fading signal at the threshold value of the ideal threshold receiver makes it very easy to produce throughput curves when we make use of different diversity techniques.

Here, we will assume that we know the embedded element efficiencies on all ports of the receive MIMO array from pure antenna simulations or measurements, as well as the correlation between its ports in a rich isotropic multipath environment. Thus, the correlations we will use are related to correlation between antenna ports in rich isotropic multipath. To produce throughput results with our new theory we will do the following: We first produce uncorrelated channel matrices numerically by using a random number generator, and include embedded element antenna efficiencies and correlations to them by multiplying with the Cholesky decomposition of the covariance matrix [13], used e.g. in [7]. These channels are then used with Maximum Ratio Combining (MRC) in (5), and finally the diversity-combined power values are arranged from the highest to the lowest values in order to produce the CCDF, and the throughput is computed using (4).

We will in the present paper only test the threshold receiver model for the simplest MIMO diversity cases, i.e. for $1 \mathrm{x} 2$ SIMO case (receiving antenna diversity) and 2x1 MISO case (transmit antenna diversity). In the latter case we assume that the transmitted power is divided equally between the antenna ports (as well as between the frequency ports) on the transmitting side (thus channel state information is not known on transmitting side).

We also choose a simplified model for the OFDM frequency diversity. This includes the OFDM bandwidth $B_{f d}$ and the coherence bandwidth $B_{c}$ (defined from complex correlation equal to 0.5 , see [10]), and assumes that the OFDM ideally corresponds to using $N_{f d}$ independent MRC-combined diversity channels, where $N_{f d}=N I N T\left(C B_{f d} / B_{c}\right)$ with NINT a function taking the nearest integer and $C$ a constant to be determined. This assumption seems to be reasonable on the background that many subcarriers and blocks covering $B_{f d}=10$ $\mathrm{MHz}$ are used to create adaptive frequency diversity, and only those parts, which are uncorrelated, can contribute to diversity.

Thus, we will use the following channel combination algorithm based on MRC, for $1 \times 2$ SIMO and $2 \times 1$ MISO cases:

$$
P_{c}=\frac{1}{N_{t} N_{f d}} \sum_{i f=1}^{N_{f d}}\left[P_{1, i f}+P_{2, i f}\right]
$$


where $N_{t}$ is the number of transmit antennas. Note that first index of power is for either two receiving or two transmitting antenna ports, and second is for frequency diversity.

\section{Measured Throughrut}

The measurement setup for throughput data rate shown in Figure 1 was used to test a commercial Huawei E398 LTE USB modem using external antennas. The USB modem was located in a separate shielded box outside the reverberation chamber. Thereby, we could connect it with separate antennas located inside the reverberation chamber, by means of two cables, and at the same time being sure that the modem's built-in antenna did not contribute to the received signal. The reverberation chamber is continuously stirred Bluetest RTS60 system loaded to achieve RMS delay spread of $90 \mathrm{~ns}$ corresponding to $3 \mathrm{MHz}$ coherence bandwidth [10]. Chamber Q was $2655 / 3=880$ and average $\mathrm{K}$-factor as defined in [14] is about 0.15 . A commercial communication test instrument was used as base station simulator and measurements were performed on LTE band 7, channel 3100 (2655 MHz). The fixed modulation of the base station was 64QAM with a bandwidth of $10 \mathrm{MHz}$, i.e. $24 \mathrm{Mbps}$ for 2x1 and 1x2 MIMO system. Note the two cables connected to the base station emulator. One is for the coinciding transmit and receive ports of the base station simulator, and the other is used by the instrument to transmit a diversity signal in the $2 \times 1$ MISO case.

\section{A. Conductive static measurements}

First we performed what is commonly referred to as conductive measurements, i.e., we measured the throughput with the two cables from the base station simulator connected directly to the two antenna ports of the LTE device. In this case we have only two antenna channels with constant power level. The measured throughput is shown in Figure 1 for the $2 \times 1$ MISO and $1 \times 2$ SIMO cases, as produced by the base station simulator instrument. The graph shows for the SIMO case also curves when we connected coaxial attenuators of different values on the two ports (the curve legends show the corresponding efficiencies). From these curves we can read the threshold value which is $P_{t}=-83.7 \mathrm{dBm}$ for both the two MISO and SIMO cases. We have used this threshold value to produce the theoretical curves in the figure by using (5) with constant power levels and the different attenuations on the ports. The threshold value has been corrected for the cable losses between the base station simulator and the antenna ports of the USB dongle. We see clearly from the figure that the measured throughput looks like that of an ideal threshold receiver with a very steep transition from zero to maximum throughput. We also see that the theoretical MRC combination scheme in (5) seem to model the different port attenuations well, except for the case of the largest amplitude imbalance of $20 \mathrm{~dB}$, for which we observe a discrepancy of about $0.6 \mathrm{~dB}$.

\section{B. Dynamic fading using reverberation chamber}

Then, we connected two separate wideband high-efficiency low-gain disk-cone antennas to the USB dongle instead of the base station simulator, using the same cables, and we connected the two ports of the base station simulator to two of the fixed antennas in the reverberation chamber, as shown in Figure 1. The two antennas are mounted orthogonal to each other with sufficient distance so that their mutual coupling and correlation become negligible. The throughput results are presented in Figure 2 where we have calibrated away the average power transfer function of the reverberation chamber including cables to the base station simulator and from the wideband antennas to USB device as well as the radiation efficiency including mismatch factor of the antennas.

In this case we have used the theoretical model exactly as explained in Section IV with Rayleigh faded channels using uncorrelated frequency channels, and combined them with MRC using (5) with $N_{f d}=2$. We have used the same value of the threshold $P_{t}$ as for the theory of the conductive static case.

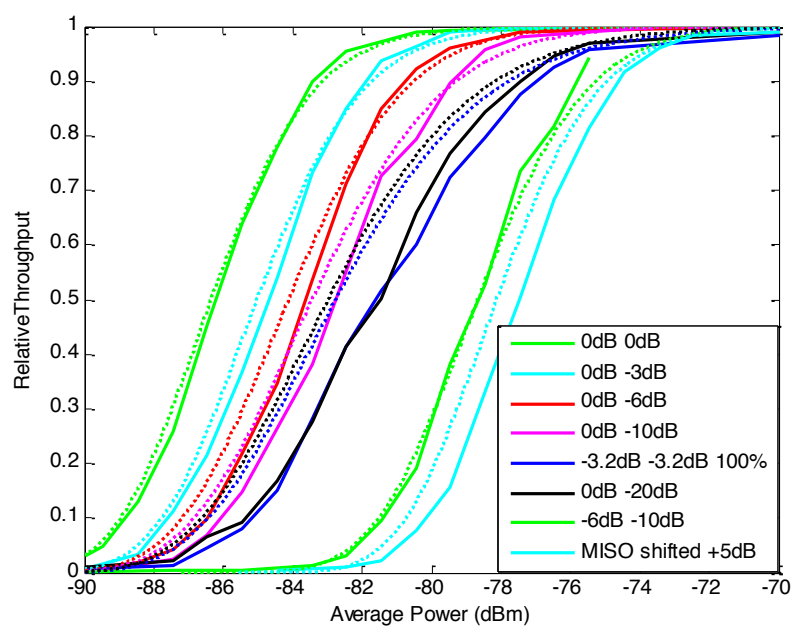

Figure 2. Measured (solid lines) and theoretical (dashed lines) relative throughput data rate for different cases of amplitude imbalance and correlation between the two receiving ports of commercial LTE USB device, for a dynamic Rayleigh fading case, for one MISO and several SIMO cases.

Figure 2 shows both the $1 \times 2$ SIMO and 2x1 MISO cases. The latter is one case only and has been moved $5 \mathrm{~dB}$ to the right for clarity. We have also now connected attenuators to the ports of the dongle in order to produce results for different amplitude imbalances for the SIMO case. We see that the theoretical model agrees very well with measured results for amplitude imbalances up to $10 \mathrm{~dB}$ on the ports. We have also produced a case of $100 \%$ correlation, by using only one of the wideband antennas, terminating the other in $50 \mathrm{ohm}$, and connecting a 2-way power divider between the first antenna and the two ports of the USB device. The $3 \mathrm{~dB}$ power divider has an insertion loss of $-3.2 \mathrm{~dB}$, which is included as an efficiency factor on both ports. We see that there is a discrepancy of $0.6 \mathrm{~dB}$ between theory and measurements both for the $100 \%$ correlated and $20 \mathrm{~dB}$ imbalanced cases. The latter is the same that we saw for conductive measurements, so it would vanish if we change the threshold value accordingly.

We have created an additional Figure 3 in order to see the theoretical effect of the OFDM frequency diversity. We found that when we include frequency diversity with $N_{f d}=$ $\operatorname{NINT}\left(C B_{f d} / B_{c}\right)=2$, i.e. $C=0.75$, the theoretical curve becomes almost identical to the measured one. 


\section{CONCLUSIONS}

In this paper we have presented a simple model of the throughput data rate for wireless devices in LTE systems with MIMO and OFDM, by which we can explain the absolute power levels of the measured throughput curve from the threshold of an ideal error correction receiver under LOS conditions. We can also explain the slope of the throughput curve from the frequency diversity achieved by the OFDM, and from using transmit $2 \times 1$ or $1 \times 2$ receive diversity in MIMO case. The simple theoretical model gives results that agree very well with the measured ones except for minor discrepancies between the slopes of the curves for the cases of 6 and $10 \mathrm{~dB}$ amplitude imbalance, and a $0.6 \mathrm{~dB}$ relative shift of the curves for the case of two $100 \%$ correlated SIMO channels and $20 \mathrm{~dB}$ amplitude imbalance. The latter is present also for conductive static case, which could be related to a change of the diversity combination algorithm (and thus threshold value) used in the USB dongle for these cases.

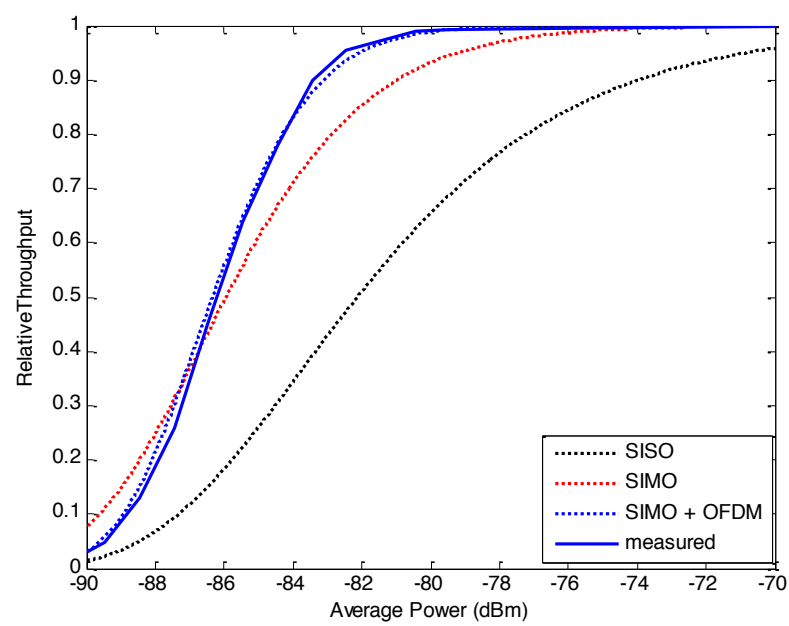

Figure 3. Theoretical (dashed lines) relative throughput data rate showing the effects on the throughput curve when we change from one receive antenna to two receive antennas and finally include frequency diversity.

The simple OFDM model seems also to work well. This means that the $10 \mathrm{MHz}$ OFDM bandwidth creates the equivalent of 2 uncorrelated frequency channels in an environment with $3 \mathrm{MHz}$ coherence bandwidth. We also loaded the chamber more to achieve $9 \mathrm{MHz}$ coherence bandwidth. Then, the slope of the throughput curve changed to agree with a theoretical curve for $N_{f d}=1$ in (5). We recalibrated the chamber transfer function because of the new load situation, but still there was a shift of about $1 \mathrm{~dB}$ between the theoretical and measured curves, even though the slopes were the same, and this needs further investigation. However, it is known that the uncertainty increases when the chamber is loaded [15], so this could very well be the reason.

Finally, it should be pointed out that similar threshold approximations to advanced digital receivers have been used in [16], We should also point out that the threshold level of the introduced threshold receiver will depend on modulation and MIMO configuration, but it is believed that these relations are simple and easily can be sorted out, see also [16] and derivative works thereof. Thus, in a real-life system with adaptive coding and Hybrid Automatic Repeat Request (HARQ) like LTE the threshold level will vary with time. Still, the ideal threshold receiver will be very important in order to model and understand OTA test situations because then the data rate and modulation are determined by the user via the settings of the communication test instrument

\section{REFERENCES}

[1] P. S. Kildal and K. Rosengren, "Correlation and capacity of MIMO systems and mutual coupling, radiation efficiency, and diversity gain of their antennas: simulations and measurements in a reverberation chamber," Communications Magazine, IEEE, vol. 42, pp. 104-112, 2004.

[2] D. A. Hill, M. T. Ma, A. R. Ondrejka, B. F. Riddle, M. L. Crawford, and R. T. Johnk, "Aperture excitation of electrically large, lossy cavities," Electromagnetic Compatibility, IEEE Transactions on, vol. 36, pp. 169$178,1994$.

[3] P. S. Kildal and K. Rosengren, "Electromagnetic analysis of effective and apparent diversity gain of two parallel dipoles," Antennas and Wireless Propagation Letters, IEEE, vol. 2, pp. 9-13, 2003.

[4] K. Rosengren and P. S. Kildal, "Radiation efficiency, correlation, diversity gain and capacity of a six-monopole antenna array for a MIMO system: theory, simulation and measurement in reverberation chamber," Microwaves, Antennas and Propagation, IEE Proceedings -, vol. 152, pp. 7-16, 2005.

[5] C. Orlenius, P. S. Kildal, and G. Poilasne, "Measurements of total isotropic sensitivity and average fading sensitivity of CDMA phones in reverberation chamber," 2005 IEEE Antennas and Propagation Society International Symposium, 3-8 July 2005, vol. Vol. 1A, pp. 409-12, 2005.

[6] A. Skårbratt, J. Åsberg, and C. Orlenius, "Over-the-Air Performance Testing of Wireless Terminals by Data Throughput Measurements in Reverberation Chamber," in 2011 European Conference on Antennas and Propagation (EuCAP2011), Rome, 2011.

[7] X. Chen, P. S. Kildal, and J. Carlsson, "Fast converging measurement of MRC diversity gain in reverberation chamber using covarianceeigenvalue approach," accepted for publication in IEICE Transactions on Electronics, 2011.

[8] N. Jamaly, P. S. Kildal, and J. Carlsson, "Compact Formulas for Diversity Gain of Two-Port Antennas," Antennas and Wireless Propagation Letters, IEEE, vol. 9, pp. 970-973, 2010.

[9] X. Chen, P. S. Kildal, J. Carlsson, and J. Yang, "Comparison of Ergodic Capacities From Wideband MIMO Antenna Measurements in Reverberation Chamber and Anechoic Chamber," Antennas and Wireless Propagation Letters, IEEE, vol. 10, pp. 446-449, 2011.

[10] X. Chen, P. S. Kildal, C. Orlenius, and J. Carlsson, "Channel Sounding of Loaded Reverberation Chamber for Over-the-Air Testing of Wireless Devices: Coherence Bandwidth Versus Average Mode Bandwidth and Delay Spread," Antennas and Wireless Propagation Letters, IEEE, vol. 8, pp. 678-681, 2009.

[11] O. Delangre, P. De Doncker, M. Lienard, and P. Degauque, "Delay spread and coherence bandwidth in reverberation chamber," Electronics Letters, vol. 44, pp. 328-329, 2008.

[12] E. Genender, C. L. Holloway, K. A. Remley, J. M. Ladbury, G. Koepke, and H. Garbe, "Simulating the Multipath Channel With a Reverberation Chamber: Application to Bit Error Rate Measurements," Electromagnetic Compatibility, IEEE Transactions on, vol. 52, pp. 766777, 2010.

[13] A. J. Laub, Matrix analysis for scientists and engineers: Siam, 2005.

[14] X. Chen, P.-S. Kildal, and S.-H. Lai, "Estimation of average Rician Kfactor and average mode bandwidth in loaded reverberation chamber," submitted to IEEE Antennas and Wireless Propagation Letters, August 2011.

[15] P. S. Kildal, L. Sz-Hau, and C. Xiaoming, "Direct coupling as a residual error contribution during OTA measurements of wireless devices in reverberation chamber," in Antennas and Propagation Society International Symposium, 2009. APSURSI '09. IEEE, 2009, pp. 1-4.

[16] A. Toyserkani, E. Strom, and A. Svensson, "An analytical approximation to the block error rate in Nakagami-m non-selective block fading channels," Wireless Communications, IEEE Transactions on, vol. 9, pp. 1543-1546, 2010. 
